Wright State University

From the SelectedWorks of Thomas Listerman

May 1, 1985

Determining the Temperature-Dependent Characteristic Temperature of Beryllium from Electrical Resistance Measurements

Thomas W. Listerman, Wright State University

Xiao-Li Zhou 


\title{
Determining the temperature-dependent characteristic temperature of beryllium from electrical resistance measurements
}

\author{
Thomas W. Listerman and Xiao-Li Zhou \\ Wright State University, Dayton, Ohio 45435
}

(Received 5 March 1984; accepted for publication 7 May 1984)

\begin{abstract}
We have developed an intermediate-level laboratory experiment to determine the temperaturedependent characteristic temperature of beryllium. The apparatus used to measure the resistance of a beryllium wire sample between liquid nitrogen and room temperatures was simple. The characteristic temperatures obtained from these data using the Block-Grüneisen model are in reasonable agreement with literature values obtained from resistivity and heat capacity experiments. The experiment introduced students to cryogenic and computer data analysis techniques and forced them to extend their knowledge of the theory of electrical resistance and of characteristic temperatures.
\end{abstract}

\section{INTRODUCTION}

After completing a typical introductory physics course, most students are familiar with the concept of electrical resistivity. They know how it is related to electrical resistance and how it might be determined for simple wire samples. Many also know about the variation of the resistivity of metals with temperature near room temperature, and some have verified this dependence in an introductory laboratory experience.

Why then might an electrical resistivity experiment be of value to intermediate-level undergraduate students? First of all, this experiment could introduce students to simple cryogenic, electrical measuring and data analysis techniques. Second, the nonlinear behavior of the resistancetemperature relationship at low temperature might be observed. Third, such concepts as Matthiessen's Rule, ${ }^{1}$ characteristic temperatures, ${ }^{1}$ and the Bloch-Grüneisen model of electron-phonon scattering ${ }^{1}$ might be applied directly in data analysis.

J. F. Thomas and R. Deltour ${ }^{2}$ have reported on such an experiment for undergraduate students. They used platinum wire samples and measured electrical resistivity between liquid helium and room temperatures. We decided not to use this experiment, however, for two reasons. First, liquid helium is not available at our university for use in undergraduate laboratory experiments. Second, the data analysis procedure presented did not lead the students to determine a characteristic Debye-like temperature for the sample and its variation with temperature. This second reason was particularly important since characteristic temperatures are discussed in modern physics, statistical physics, and solid state physics courses, yet are never actually determined by students in their undergraduate laboratory experiments.

In order to replace the liquid helium coolant with more readily available liquid nitrogen, we used the Bloch-Grüneisen model to find a better sample. This model predicts that for $T / \theta$ below 0.2 the resistivity should be strongly nonlinear. ${ }^{3}$ Assuming for now the characteristic temperature is essentially equal to the Debye temperature, this criterion tells us that a sample must have a Debye temperature greater than $390 \mathrm{~K}$ to see any nonlinear effects near liquid nitrogen temperatures. Since the nonlinearity increases as $T / \theta$ decreases, we should select a sample with as large a Debye temperature as possible. Beryllium, with a Debye temperature of $1390 \mathrm{~K}$ at low temperatures, ${ }^{4}$ has the lar- gest Debye temperature of the metallic elements and is also readily available in wire form. It was the obvious choice. (An interesting exercise for students is to figure out for themselves why beryllium is a good choice and to evaluate the suitability of other materials.)

\section{EXPERIMENT}

The apparatus used for holding the sample and controlling its temperature was particularly simple and is shown in Fig. 1. The core of the sample holder was a cylinder of brass (A in Fig. 1) measuring $5 \mathrm{~cm}$ long and $2.5 \mathrm{~cm}$ in diameter though these dimensions are not at all critical. This was chosen for three reasons: Brass has a fairly high thermal conductivity and the cylinder should therefore have a fairly constant temperature throughout its volume; the heat capacity of the cylinder was rather large which provided thermal inertia against rapid temperature changes; and this particular piece happened to be available in our scrap metal bin. Its curved outer surface was wrapped with a single layer of mending tape ${ }^{5}$ to provide electrical insulation with only small thermal insulation for the sample. The beryllium wire sample was wrapped over this tape, and a second layer of tape was wrapped around the outside to hold the sample in place. Both ends of the sample protruded from the wrapping and were attracted to copper leads with small brass screws and nuts. A thermocouple was also taped to the outside of the cylinder to measure the temperature of the sample-sample holder combination.

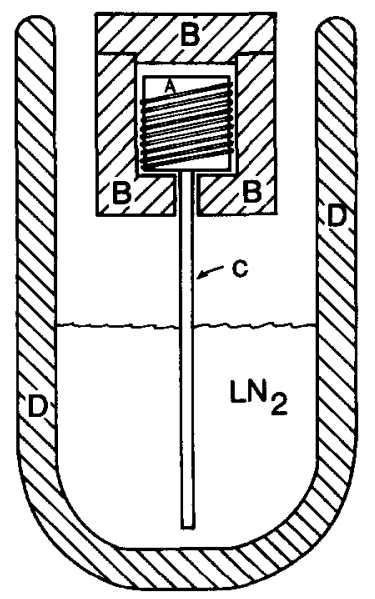

Fig. 1. Experimental apparatus. 
The sample holder was enclosed in a home-made styrofoam container for thermal insulation (B in Fig.1). A long brass rod (C in Fig. 1) was threaded into the cylinder on one end and extended through a hole in the styrofoam to transfer heat from the sample holder to the liquid nitrogen bath. Electrical leads and thermocouple wires were brought out through this same hole. This thermally insulated sample holder was suspended within a glass dewar ( $D$ in the diagram) with the brass rod extending down to near the bottom of the dewar.

The samples were $100-\mathrm{cm}$-long, 0.25 -mm-diam beryllium wires purchased from Alfa Products. ${ }^{6}$ The purity specification was $99.5 \%$ for metallic impurities and $99+\%$ for total impurities. B. W. Powell ${ }^{7}$ found that annealing beryllium samples near $1000^{\circ} \mathrm{C}$ was required to get useful reproducible results. We did not do this with out samples because of the toxicity of beryllum vapor and because this did not seem necessary for an undergraduate laboratory experiment.

Four copper leads and two thermocouple wires, all small in diameter, extended out of the dewar. Two of the copper leads brought current to and from the sample and were connected in series with a standard resistor and a power supply. Currents of the order of $4 \mathrm{~mA}$ were used during the experiment to reduce self-heating in the sample and to insure that the sample temperature was essentially that of the sample holder. The other two copper leads were used to measure the voltage drop across the sample. The reference junction of the thermocouple was placed in an ice-water bath. The thermocouple wires, the voltage leads connected across the sample, and the leads connected across a standard resistor were connected through two DPDT switches to a potentiometer so that the potential drop across each pair of leads could be separately measured. The sample temperature was determined from the measured thermocouple emf using standard tables. ${ }^{8}$ The sample resistance was determined using Ohm's law and the measured potential drops across the sample and standard resistance. ${ }^{9}$

The experimental procedure consisted of placing some liquid nitrogen in the dewar with the sample holder in place and measuring resistance and temperature "on the run" as the sample cooled. Since the complete cooling took $2-3 \mathrm{~h}$, this simple procedure worked well.

\section{RESULTS}

The unrefined resistance-temperature data obtained by several different student groups in an intermediate-level lab course on two different beryllium wire samples are shown in Fig. 2. This clearly shows that the two samples had a slightly different resistance-temperature characteristic. Since this experiment was a teaching laboratory exercise rather than a research project, we made no effort to remove these differences by heat treatment as mentioned before. The samples also became more brittle after several coolings which resulted in broken lead connections when the sample was handled roughly. No effects of this hardening were observed in the data, however. These results are clearly nonlinear at lower temperatures as expected. The low-temperature data appear to converge to a positive value of resistance in the limit as $T$ approaches $0 \mathrm{~K}$ as described by Matthiessen's Rule. At the highest temperatures, the results approach linearity, which is the expected high-temperature behavior.

Quantitative analysis of these data correlates with these

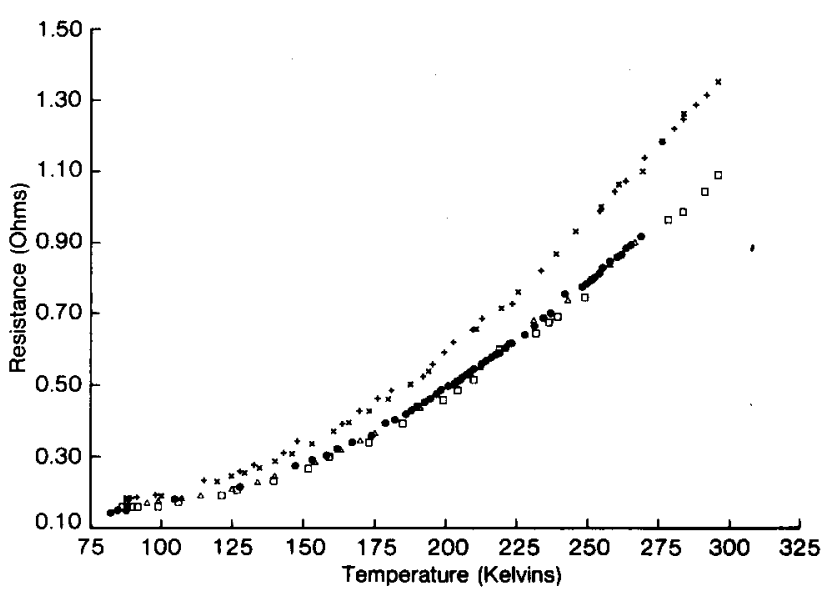

Fig. 2. Resistance-temperature data.

observations. Since our primary interest was in the temperature dependence of resistivity rather than resistivity values per se, we analyzed resistance data directly rather than compute resistivity values first. We also made no corrections for thermal expansion of the sample.

The first step was to apply Matthiessen's Rule

$$
R=R_{0}+R(T)
$$

to separate the temperature-independent resistance contribution $R_{0}$ attributed to impurity scattering and the temperature-dependent contribution $R(T)$ attributed to phonon scattering. This was first done graphically by extrapolating the $R$ vs $T$ curve back to $0 \mathrm{~K}$, but this extrapolation was very uncertain. Fortunately Reich et al. ${ }^{10} \mathrm{ob}-$ served that the resistivity of beryllium was proportional to $T^{4}$ between $50 \mathrm{~K}$ and $130 \mathrm{~K}$. This meant that

$$
R=R_{0}+b T^{4}
$$

and that a plot of $R$ vs $T^{4}$ should yield a straight, and easily extrapolated, line. Our data followed such a relationship, and values of $R_{0}$ were obtained graphically from these plots. We also used the linear regression subroutine programmed in a hand-held calculator to least-squares fit our data to this linearized curve and then used $R_{0}$ values from this fit in all succeeding calculations.

Once $R_{0}$ was subtracted from measured resistance values, the resulting temperature dependent resistance component could be analyzed in terms of microscopic models of the scattering processes. The Bloch-Grüneisen model ${ }^{1}$ is particularly useful due to its relative simplicity and accuracy and to the fact that it yields a characteristic temperature roughly comparable to the Debye temperature. The approximations and limitations of this model are discussed in many standard references. ${ }^{1}$ This model predicts that the temperature-dependent resistance component is given by the relationship ${ }^{1}$

$$
R(T)=\left(K T / \theta^{2}\right) G(\theta / T),
$$

where

$$
G(\theta / T)=4\left(\frac{T}{\theta}\right)^{4} \int_{0}^{\theta / T} \frac{x^{5}}{\left(e^{x}-1\right)} \frac{d x}{\left(1-e^{-x}\right)} .
$$

In these equations $T$ is the sample temperature, and $\theta$ is the characteristic temperature, both in Kelvins, and $K$ is a product of several constants and a factor characterizing the electron-phonon interaction. We considered $K$ to be inde- 
pendent of temperature which is a standard assumption in this type of analysis. ${ }^{11}$

According to the assumptions of this model, $\theta$ is a constant which characterizes the sample and should be identical to the Debye temperature. At high temperatures $(T \gg \theta)$ the model predicts that $R(T) \propto T$ and at low temperatures $(T \ll \theta)$ the model predicts that $R(T) \propto T^{5}$. At intermediate temperatures the definite integral in $G(\theta / T)$ must be evaluated. This does not yield a simple analytical form, and numerical tables of values of $G(\theta / T)$ are required. Due to beryllium's high characteristic temperature, the highest temperatures encountered in our experiment are only about $\theta / 4$, and none of our data corresponds to the hightemperature limiting case. At low temperatures the resistance of beryllium is proportional to $T^{4}$ as previously mentioned and not to $T^{5}$. At intermediate temperatures the data also do not follow the model exactly although they are "in the right ballpark." The same sort of problems are encountered in applying the Debye theory to heat capacity data at intermediate temperatures, and the same "solution" is applied in both cases. Rather than assuming that $\theta$ is constant, a temperature-dependent characteristic temperature is introduced and tabulated. At any given temperature, values of resistance or specific heat may be computed using these models by inserting the appropriate value of the characteristic temperature for the sample temperature in question. We have determined the temperature-dependent characteristic temperature for each data set shown in Fig. 2.

Kelly and MacDonald ${ }^{12}$ have described four principal methods for determining $\theta$ values from resistivity data; these methods are also discussed by Meaden. ${ }^{13}$ We used the first two methods which are relatively straightforward applications of the theory. With both of these methods, tables of values of $G(\theta / T)$ are required. Meaden ${ }^{13}$ provides such tables although we computed more extensive tables for our use. Using the trapezoidal rule for numerical integration, ${ }^{14}$ we tabulated values of $G(\theta / T)$ for $\theta / T$ values ranging from 0.0 to 80.0 in steps of 0.1 . Values of $G(\theta / T)$ for intermediate values of $\theta / T$ were found by linear interpolation between these tabular values.

The first method allows us the determine a single $\theta$ value consistent with each pair of data points. Equation (3) is first evaluated for each of the data points assuming $\theta_{1}=\theta_{2}$. The resulting two equations are then solved simultaneously to elimate $K$, assumed to be constant, which yields

$$
G\left(\theta / T_{1}\right)=\frac{R_{1}}{R_{2}} \frac{T_{2}}{T_{1}} G\left(\theta / T_{2}\right) .
$$

In this equation $R$ refers to a temperature-dependent resistance component, $T$ refers to a temperature, and the subscripts 1 and 2 refer to the two data points being analyzed. $\theta$ values are determined by substituting trial values of $\theta$ into Eq. (5) until the left and right sides of the equation are equal.

We first determined by hand the $\theta$ values for several pairs of data points. This procedure was tedious but was useful in understanding the method. We then wrote a computer program which searched for $\theta$ values that made the two sides of Eq. (3) equal, within a specified tolerance, using a binary search technique. ${ }^{14}$ This allowed us to determine $\theta$ values for every pair of data points in each data set in a very short time. The results showed roughly constant $\theta$ values for pairs of high-temperature points and lower $\theta$ values for combinations of a high-temperature and a low-tempera- ture point. A good deal of scatter was also present due to experimental error.

These results are difficult to use, however, if $\theta$ is a very strong function of temperature. We want to find the $\theta$ value for a single temperature, not the $\theta$ value consistent with pairs of data points at two different, and perhaps widely spaced, temperatures. The real value of this method is in finding $\theta$ values in cases where $\theta$ is expected to be practically constant over a range of temperatures.

The second method allows us to determine the $\theta$ value at one temperature if the $\theta$ value at a second temperature is known. As in the first method, Eq. (3) is evaluated for each of the data points and these two equations solved simultaneously to eliminate $K$ and yield

$$
\left(\frac{T_{1}}{\theta_{1}}\right)^{2} G\left(\theta_{1} / T_{1}\right)=T_{1} R_{1}\left[\frac{T_{2}}{\theta_{2}^{2} R_{2}} G\left(\theta_{2} / T_{2}\right)\right] .
$$

The symbols are the same as in Eq. (5) except that subscript 2 refers to the temperature at which we know the $\theta$ value, $\theta_{2}$ is the known $\theta$ value, and $\theta_{1}$ is the $\theta$ value to be determined. $\theta_{1}$ values are determined by substituting trial $\theta_{1}$ values into Eq. (6) until the left- and right-hand sides are equal.

In order to use this method, we first had to determine a known $\theta$ value. Since our results using the first method showed roughly constant $\theta$ values for pairs of high-temperature points, we first decided to use the average of the $\theta$ values for all of the pairs of data points in the data set above $250 \mathrm{~K}$. This $\theta$ value would then be assigned to the temperature at the midpoint of the high-temperature range, typically $275 \mathrm{~K}$. The resistance at this temperature would be computed from a least-squares fit of the high-temperature data to a straight line. These would be substituted into Eq. (6) as $\theta_{2}, T_{2}$, and $R_{2}$, and for each of the data points in the data set, a $\theta_{1}$ value would be determined. As before, this would be carried out by a computer using a binary search technique. ${ }^{15}$ The only flaw in this procedure seemed to be the large scatter of the high-temperature $\theta$ values from the average. Smali experimental errors in the pairs of data points used to find $\theta$ values led to fluctuations as large as $15 \%$ of the average.

Due to these large fluctuations, we decided instead to use the second method itself to determine known $\theta$ values subject to the requirement that $\theta$ vary as little as possible at temperatures greater than $250 \mathrm{~K}$. We used $T_{2}$ and $R_{2}$ values obtained as previously described, assumed a $\theta_{2}$ value, and for each data point above $250 \mathrm{~K}$ found a $\theta$ value as described before. We computed the variance of these $\theta$ values from their average, incremented the assumed $\theta_{2}$ value, and repeated the procedure. This continued until a $\theta_{2}$ value giving the minimum variance was found, and this $\theta_{2}$ value was assigned to the high-temperature midpoint. This value typically was within $1 \%$ of the $\theta_{2}$ value determined previously by simple averaging. All of the points in the data set were then analyzed using this $\theta_{2}$ value. This procedure was then repeated for each data set.

The resulting temperature-dependent $\theta$ values determined by this method, for each of the sets of student-measured data reported in Fig. 2, are given in Fig. 3. Each data set is represented by the same symbol in Fig. 2 and 3. The results for the two samples are again different, as in Fig. 2. The lower resistance sample yields higher $\theta$ values although the separation is not as distinct as in Fig. 2. As is the case for Debye temperatures determined from specific heat measurements, ${ }^{15} \theta$ is constant at higher temperatures but 


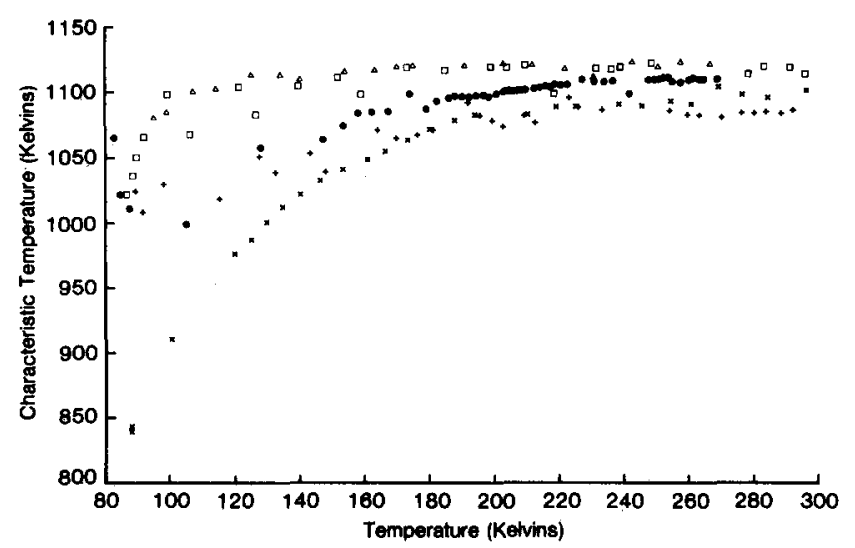

Fig. 3. Characteristic temperatures as a function of temperature.

drops at lower temperatures. $\theta$ values also agree with the wide range of values in the literature. Debye temperatures of $1160 \mathrm{~K}$ (see Ref. 16) and $1390 \mathrm{~K}$ (see Ref. 4) at $0 \mathrm{~K}$ range to $940 \mathrm{~K}$ (see Ref. 17) and $1000 \mathrm{~K}$ (see Ref 4) at $\theta_{D} / 2$. Characteristic temperatures obtained from resistivity measurements at higher temperatures range from $652 \mathrm{~K}$ (see Ref. 16) to $1240 \mathrm{~K}$ (see Ref. 10). Our values of roughly 1100 $\mathrm{K}$ for unannealed polycrystalline specimens are certainly in the right range. We attribute the difference between the results of successive experiments on the same sample to student errors. These data were obtained by students during their first and only experimental run and do not include any refinements of procedure or technique resulting from multiple runs and analyses.

\section{CONCLUSIONS}

We concluded that this experiment was successful in exposing students to new experimental techniques including computer data analysis techniques. Hand calculations were useful in gaining a better understanding of the data reduction procedures. Additional study of solid-state physics concepts, beyond that considered in formal classes, was required to understand the experiment and to write an adequate report. Since the required apparatus is simple and since liquid nitrogen and computer facilities are commonly available today, this experiment could be implemented rather easily at many colleges. ${ }^{18}$

\section{ACKNOWLEDGMENTS}

We wish to thank Abraham Atachbarian, Richard Furlan, Peggy Grigsby, Christopher Johnson, and Gregory Worrell whose experimental data were also used in this analysis.

\footnotetext{
'See standard reference texts such as J. M. Ziman, Electrons and Phonons (Oxford University, London, 1960); G. T. Meaden, Electrical Resistance of Metals (Plenum, New York, 1965); Charles Kittel, Introduction to Solid State Physics, Second Edition (Wiley, New York, 1956).

${ }^{2}$ J. F. Thomas and R. Deltour, Am. J. Phys. 49, 276 (1981).

${ }^{3}$ Meaden, Ref. 1, p. 81.

${ }^{4}$ Meaden, Ref. 1, p. 100.

${ }^{5}$ Highland Brand 6200 Permanent Mending Tape, Commercial Tape Division/ 3M, St. Paul, MN 55144.

'Alfa Products, Thiokol/Ventron Division, P. O. Box 299, Danvers, MA 01923.

${ }^{7}$ R. W. Powell, Philos. Mag. 44, 645 (1953).

${ }^{8}$ Omega Temperature Measuring Handbook (Omega Engineering, Inc., Stamford, CT, 1975).

${ }^{9}$ This and other measuring techniques are discussed in Meaden, Ref. 1, Chap. 7.

${ }^{10}$ R. Reich, V. Q. Kinh, and J. Bonmarin, C. R. Acad. Sci. (Paris) 256, 5558 (1963).

${ }^{11}$ Meaden, Ref. 1, Sec. 4.3

${ }^{12}$ F. M. Kelly and D. K. C. MacDonald, Can. J. Phys. 31, 147 (1953).

${ }^{13}$ Meaden, Ref. 1, Chap. 4.

${ }^{14} \mathrm{~F}$. Scheid, Computers and Programming (McGraw-Hill, New York, 1982), pp. 250-254.

${ }^{15}$ E. S. R. Gopal, Specific Heats at Low Temperatures (Plenum, New York, 1966), p. 36.

${ }^{16}$ Ziman, Ref. 1, p. 366.

${ }^{17}$ Gopal, Ref. 15, p. 33.

${ }^{18}$ Copies of FORTRAN programs used in this analysis may be obtained from T. Listerman.
}

\title{
Scattering approach to Fresnel's equations and Brewster's law
}

\author{
William T. Doyle
}

Physics Department, Dartmouth College, Hanover, New Hampshire 03755

(Received 2 April 1984; accepted for publication 29 May 1984)

\begin{abstract}
A factored form of Fresnel's equations for arbitrary transparent media is presented. The two factors separately express the scattering pattern of the individual dipoles and the coherent scattering function of the dipole array. The new form facilitates comparison with the scattering interpretation of reflection and refraction. It is used to develop a new scattering model of Brewster's law.
\end{abstract}

\section{INTRODUCTION}

According to Brewster's law, light reflected from the surface of a transparent medium is completely polarized when the transmitted and reflected beams are mutually perpendicular. While Maxwell's equations, in the guise of the Fresnel coefficients, describe the effect quantitatively, the generality, simplicity, and geometrical character of 